\title{
WAY-FINDING ASSISTANCE SYSTEM FOR UNDERGROUND FACILITIES USING AUGMENTED REALITY
}

\author{
K. Yokoi ${ }^{\text {a.* }}$ N. Yabuki ${ }^{\text {a }}$ T. Fukuda ${ }^{\text {a }}$ T. Michikawa ${ }^{\text {b }}$, A. Motamedi ${ }^{\text {a }}$
${ }^{a}$ Division of Sustainable Energy and Environmental Engineering, Osaka University, Suita, Osaka, Japan yokoi@it.see.eng.osaka-u.ac.jp, (yabuki, fukuda, motamedi)@see.eng.osaka-u.ac.jp
${ }^{\mathrm{b}}$ Center for Environmental Innovation Design for Sustainability, Osaka University, Suita, Osaka, Japan michikawa@ceids.eng.osaka-u.ac.jp

WG IV/7 and WG V/4

\begin{abstract}
KEY WORDS: Augmented Reality, Structure from Motion, Way-finding, Navigation Assistance, Underground Facilities
\end{abstract}
\begin{abstract}
:
Way-finding is one of main challenges for pedestrians in large subterranean spaces with complex network of connected labyrinths. This problem is caused by the loss of their sense of directions and orientation due to the lack of landmarks that are occluded by ceilings, walls, and skyscraper. This paper introduces an assistance system for way-finding problem in large subterranean spaces using Augmented Reality (AR). It suggests displaying known landmarks that are invisible in indoor environments on tablet/handheld devices to assist users with relative positioning and indoor way-finding. The location and orientation of the users can be estimated by the indoor positioning systems and sensors available in the common tablet or smartphones devices. The constructed 3D model of a chosen landmark that is in the field of view of the handheld's camera is augmented on the camera's video feed. A prototype system has been implemented to demonstrate the efficiency of the proposed system for way-finding.
\end{abstract}

\section{INTRODUCTION}

Underground spaces play an important role in big cities. They have been mainly developed as commercial spaces in association with the development of the subways. These spaces are used not only for shopping sites, but also for commuting route and for avoiding extreme weather conditions. Indeed, many big cities have developed large underground spaces (e.g. Tokyo and Osaka in Japan).

Underground spaces pose one major problem for pedestrians that is the difficulty with finding the route to the destination, called way-finding problem. The underground spaces are usually developed incrementally and the underground spaces become complex network on connected labyrinths. Way-finding in these connected underground spaces is not only inconvenient for pedestrians but also critical in the case of emergency (e.g. flooding and fires), as the pedestrians should be evacuated from the underground spaces quickly. Regarding the evacuation behavior, Taniguchi et al. (2014) researched about the evacuating plan in case of Tsunami in Umeda underground spaces, Osaka, and simulated the situation by creating a Virtual Reality (VR) world of the underground.

The way-finding problem is mainly caused by the loss of the sense of direction and orientation due to lack of landmarks visible from a distance (such as tall buildings or city monuments), or inability to visually identify recognizable natural features (such as sun, mountains and hills). The same problem exists outdoors in urban areas where the visibility is obstructed by dense highrise buildings. The Global Positioning System (GPS) is not accurate indoors and in dense urban areas. Hence, the current method for way-finding and navigation relies only on the placement of map and signage. However, these methods have limitations and are less intuitive compared to visual way-finding methods using known landmarks.
Regarding signs, one of the issues is that the number of the destinations which are written on the signs are limited. Furubayashi et al. (2013) researched about the discontinuity of the directional sign in subway stations. They developed a mathematical data model and a system for examining whether the sign can efficiently guide pedestrians to all exits. According to their case study, there are intermittent signs as well as destinations within the station that cannot be reached by using only installed signage.

Moreover, reading maps and floor plans can be difficult for many pedestrians as they need to locate their current location on the map, figure out their orientation, locate their destination, and decide on the route to a desired the destination. Additionally, they need to constantly check the map to make sure the right path is being taken. As a result, many pedestrians may choose to go aboveground to be able to see landmarks.

This paper presents a method to assist solving the way-finding problem using augmented reality (AR). AR is a technology to combine virtual objects (CG models) and the real-time feed of video images. This enables us to enhance real world image by displaying invisible objects. Our proposed system uses this technology to display invisible landmarks to pedestrians in underground spaces. When the users direct tablet devices to desired directions, the system shows the landmarks overlaid on the camera images in real time. The landmarks are arranged based on positioning systems and sensors in the device. As the landmarks are overlaid to the real-time image, the system can facilitate reaching a desired destination by providing a positional relationship between underground spaces and above ground spaces. 


\section{RELATED WORK}

Regarding the application of AR for guidance, Yamauchi (2012) developed an AR application that shows the 3D models of each floor in a smart phone by using markers on the ceiling, which are identified by the camera. This application enables the user to understand the current location and the route to the ground level more easily and intuitively. However, in this research, users have to compare a 3D map in their devices with the real world scene. Therefore, the problems related to difficulties with map-reading are remained.

TagCast. Inc (2014) developed a navigational aid application for underground facilities. The application uses AR to show direction signs on the smartphone. It helps pedestrians navigate to their chosen destinations. However, the application only provides directions to the users and does not provide them with an overall view of the path and the location relationships between the current position and the destination.

Regarding other guidance methods, Tardieu et al. (2009) proposed an auditory way-finding system. In their research, three sound signals were used: Orientation, Confirmation, and Timeline. The first sound signal directs the users to the destination. The second sound signal confirms at each step of the walk that the direction is right. The third signal informs the users, at each step, about the progression of the walk. In the way-finding experiment with the auditory system, compared to the experiment without the system, more participants completed the steps without any difficulty. However, sound signal does not provide pedestrians with an overall view of the path and the location relationships between the current position and the destination.

Regarding research related to showing the invisible objects occluded by other objects using AR, Kameda et al. (2004) introduced a method for visualizing invisible areas that are monitored by surveillance cameras to the user. Given a 3D model of the occluded objects and video of the invisible area, the method displays the blind spots by texture mapping of the video and the $3 \mathrm{D}$ objects using semi-transparent rendering. However, this system requires the presence of video cameras in the area and the large usage of resources. Schall et al. (2009) introduced a visualization system for underground infrastructure (such as pipes) using AR. The system supports recognizing the relationship between the facilities in construction in order to avoid unexpected accidents. These research projects demonstrate the application of AR technology in efficiently visualizing invisible objects.

\section{PROPOSED SYSTEM}

\subsection{Overview of the System}

As discussed in Section 2, AR-based navigational aid systems often provide users with direction signs for guiding them within the facility. They usually do not give the user an overall view of their orientation or the relative distance to known city landmarks. However, the common walking experience includes observing natural/man-made landmarks and subconsciously tracking the relative distances to these known points. Although a pedestrian does not exactly know where he is geolocated while walking, the relative distance to these landmarks gives him/her an approximation of his location, the direction where the destination is located, and the time it takes to reach it.

As explained in Section 1, our proposed system is an ARapplication for hand-held devices. When the users point the device to a desired direction in the underground floor, the system shows live stream of images from the camera with $3 \mathrm{D}$ objects of the landmarks overlaid on them. The user can then recognize the direction to the landmarks, accordingly. Figure 1 shows an application scenario for the proposed system.

Our proposed system does not aim to provide users with direction guidance to reach specific destinations. Instead, it provides user with visual information about invisible, known landmarks. By using the proposed system, the user can enhance his way-finding experience in underground spaces or dense urban areas.

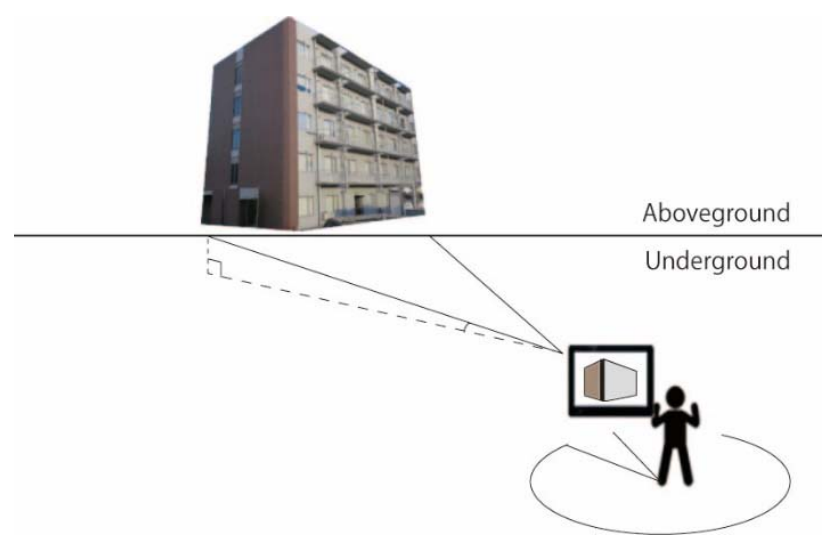

Figure 1. Application scenario

\subsection{Application Scenarios:}

Scenario 1: User aims for a specific destination: The pedestrian uses the system to get information about how far he is from the destination or if he is heading to the right direction.

Steps: (1) the 3D model of the destination is chosen from a list; (2) the 3D model is shown on the screen when the tablet is held towards its direction; (3) The size/view angle of the model is changed based on the distance and the relative location of the user to the destination; (4) The view can be accompanied by Plan Position Indicator (PPI) and navigational aid such as voice and arrow signs

Scenario 2: User is strolling or walking to an undecided destination (for example for the purpose of shopping, jogging, or sightseeing): The pedestrian uses the system to approximate how far he is from a set of known landmarks and which direction he is heading relative to known landmarks.

Steps: (1) user selects a set of landmarks; (2) the 3D model of selected landmarks are shown on the screen when the tablet is held towards their direction; (3) The size/view angle of the models are changed based on the relative distance and the angel.

In order to realize the system two major steps should be taken: (1) Preprocessing: to construct and arrange 3D models of landmarks in a virtual spaces before using the system; (2) Augmentation: to display the overlaid images on the hand-held device. These two steps are described in details in the following subsections.

\subsection{Pre-processing}

Our system requires 3D models of the landmarks to be used in the application. These objects should be modelled prior to the use of the system as a pre-processing step. Several methods for creating 3D models of the landmarks exist. 3D objects can be manually created by CAD systems (e.g. Autodesk 3ds Max and 
Revit), however, this method is labour intensive and timeconsuming. Additionally, the use of laser scanners provides us with "as-built" models automatically from point cloud data, however, the scanning devices are not usually used due to high cost.

This research uses Structure from Motion (SfM) (Dellaert et al., 2000) techniques to create 3D point cloud data of the landmarks. Using this approach, 3D objects can be easily reconstructed from a set of photographs using software applications such as Bundler (Snavely et al., 2006), OpenMVG (Moulon, 2012), and PhotoScan (Agisoft LLC., 2006). This approach has a potential to create the 3D models using available photos. These photos can be obtained from the image hosting services, such as flickr.com or any other source of images. For example, Agarwal et al. (2011) used photos in the cloud image hosting services to reconstruct historical landmarks. In this research, 150,000 photographs, which is returned when entering the search term "Rome" on flickr.com, were used. The system uses a collection of parallel distributed matching and reconstruction algorithms, designed to maximize parallelism at each stage and minimize serialization bottlenecks. Therefore, reconstructing 3D points from images took only 21 hours. The SfM approach is used in our system as it provides the desired accuracy and speed fitted to our objectives. Since the SfM does not consider scaling, the scaling factor is manually adjusted. Figure 2 shows the constructed 3D model of the building in Figure1 created using SfM technique.

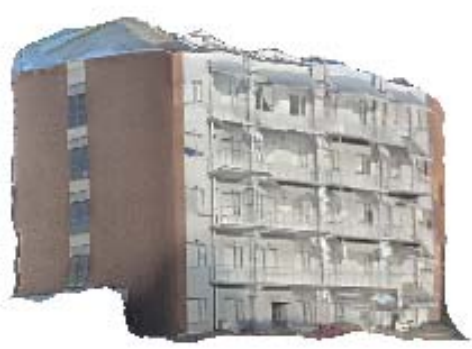

Figure 2. The model created by SfM

In our method, the landmarks need to be manually geolocated. However, there are techniques to calculate the location coordinates of the landmarks by using the GPS information embed in its photographs (Junejo and Foroosh. 2008).

Although SfM is used in our system (as it is fast, relatively accurate and high performance), other techniques to construct the 3D object for landmarks can be also used. Additionally, available 3D models in virtual map applications (such as Google Earth) or $\mathrm{CAD}$ applications can be used in our proposed system.

\subsection{Augmentation}

The main function of the system is to combine the $3 \mathrm{D}$ objects of the landmarks and the live feed from the video camera of the tablet. In order to combine them, the current position of the user is acquired from some sensors in the tablet device. For example, the position is acquired from real-time positioning systems (such as Global Positioning System (GPS), UltraWide Band (UWB), Radio Frequency Identification (RFID) and location markers) and, the orientation and its changes are also acquired from the digital compass (magnometer) and gyroscopes. As most of the current smartphones and tablet devices are equipped with these sensors, the system can be easily utilized on the common devices.
By having these information, combined images are created. For that, the information of the current position and orientation is acquired. The tablet's camera is setup. If the landmarks are in the field of view of the camera, the 3D models are overlaid on the video scene.

\section{CASE STUDY}

\subsection{Description of Prototype System}

In our prototype systems, Microsoft Windows 8.1 was used as the development environment, and Metaio SDK (Metaio Inc. 2013) was used for the AR development. The software development is done using Java programming language. For the field experiment, Sony Xperia acro So-02C android smartphone was used. In the field test, GPS technology was utilized for positioning and PhotoScan SfM software was employed to construct 3D models.

The SfM technique shows high efficiency for creating complex 3D models (such as the one on this research) due to its ability to construct the models automatically. The steps for creating the model using SfM are visualized in Figure 3 and are as follow: (1) Preparation of photos: the photos of the landmarks took from a various angle of view should be prepared, and the size of photos should be unified using photo editing software. It is also desirable to prepare aerial photograph for better results. (2) Reconstructing 3D point data from photographs; (Snavely et.al. 2006). (3) Estimating high density point data from Step 2 (Furukawa et.al. 2010). (4) Creating mesh data from Step 3. (5) Sticking the texture to the step 4's model.
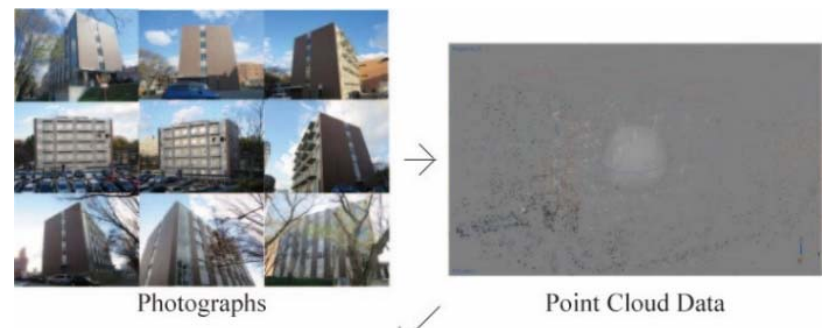

Point Cloud Data

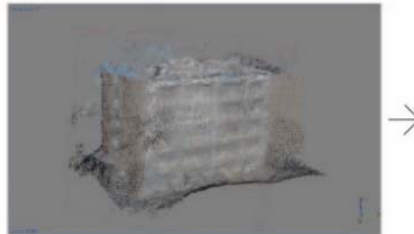

High Density Point Data

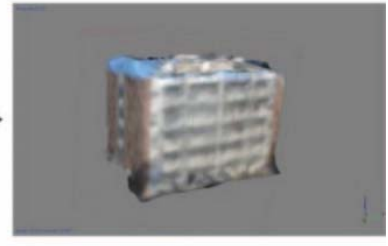

Mesh Model

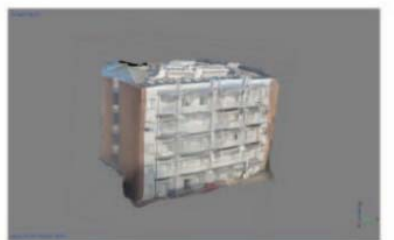

Textured Model

Figure 3. SfM Steps 


\subsection{Experiment}

The experiment was done in the basement first floor of the U1W building of Osaka University (Figure 4). The augmented landmark was set to be the model of US1 Building in the same campus (Figure 5). Figure 6 shows the relative location of the two buildings in the experiment. US1 building is situated at lat. $34^{\circ}$ $821^{\prime} \mathrm{N}$, and long. $135^{\circ} 523^{\prime} \mathrm{E}$, and elevation. $60.034 \mathrm{~m}$. U1W is situated at lat. $34^{\circ} 823^{\prime} \mathrm{N}$, and long. $135^{\circ} 520^{\prime} \mathrm{E}$, and elevation. $58.020 \mathrm{~m}$. Regarding location relationship, US1 building is located in southeast of U1W building with an approximate distance of 350 meters, as shown in Figure 6. 162 pieces of photographs were prepared as input for SfM. The pictures were then resized to 640 pixel $\times 640$ pixel. It took 5 minutes 54 seconds to finish creating the $3 \mathrm{D}$ point cloud data using our SfM software.

In our prototype application, Plan Position Indicator (PPI) were showed on the screen in order to help users understand the relative location and distance of the landmark and the direction of the device. Figure 7 and Figure 8 shows snapshots of the developed application. In these figures, the blue icon shows the location of the user, and yellow icon shows the location of the landmark (represented by the AR model). Additionally, the field of view and the visibility range is also shown.

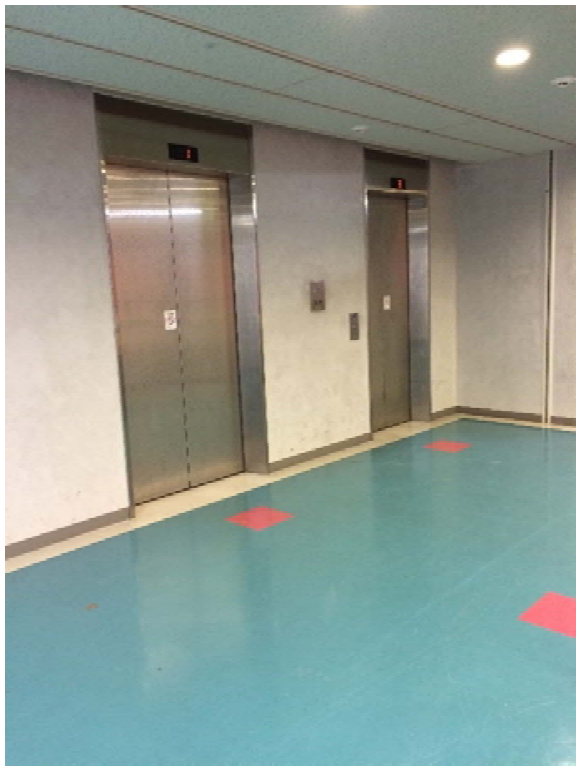

Figure 4. U1W Building Indoor Environment

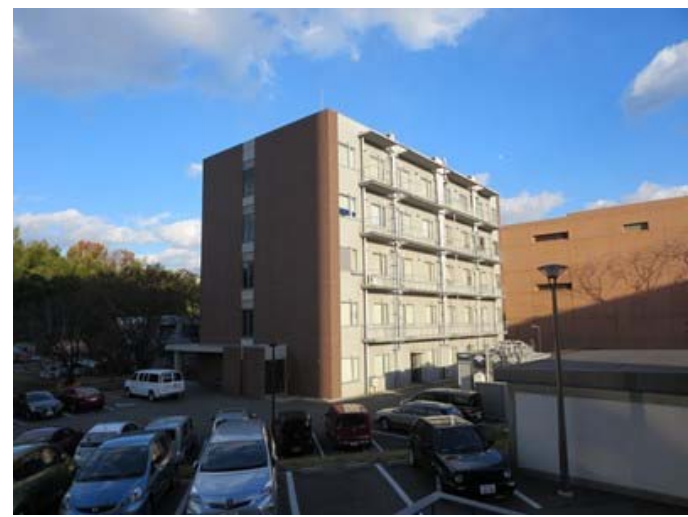

Figure 5. Osaka University US1 Building

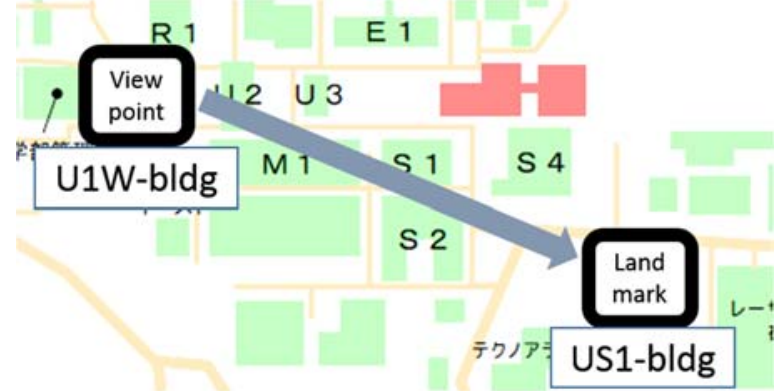

Figure 6. Location of U1W and US1

\subsection{Results}

Figure 7 shows the prototype application in action. In the camera screen, PPI view is shown at the upper left and the augmented landmark is shown in the middle. Our field test confirmed that the location of the model and the shown direction of the handheld on the PPI view are correct (Figure 8). Additionally, the test confirmed that the augmented model of US1 building is shown while directing the phone towards it.

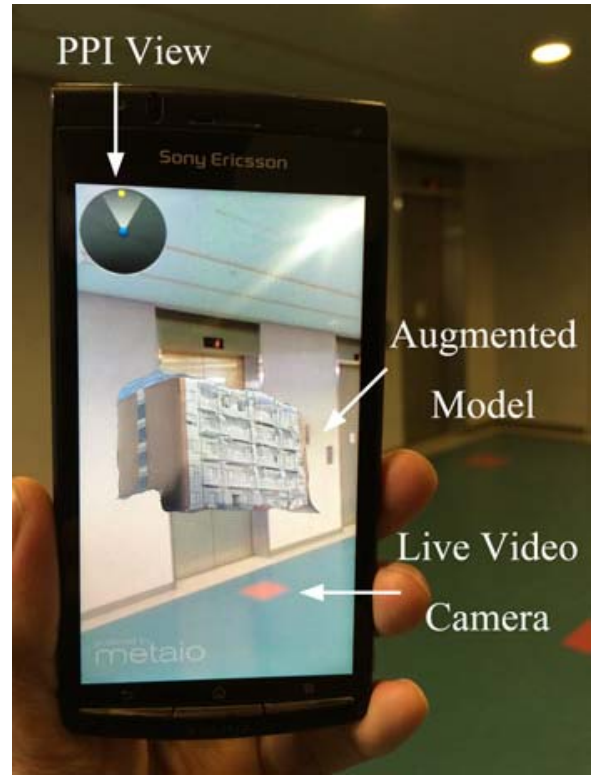

Figure 7. Snapshot of Application

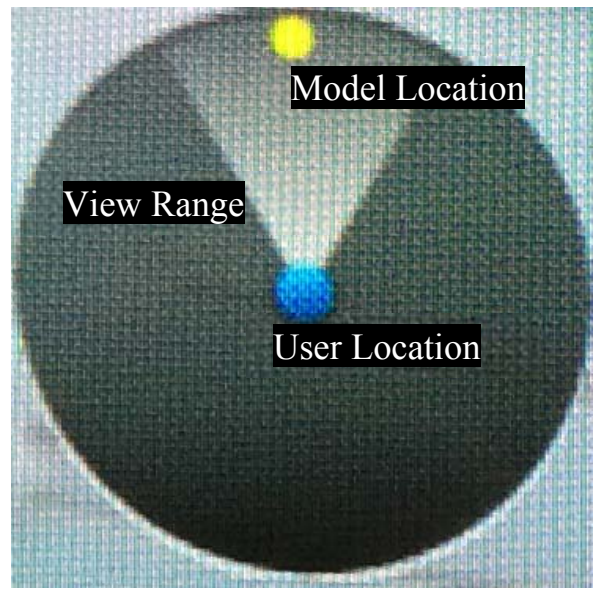

Figure 8. Plan Position Indicator 


\subsection{Discussion}

The created model for our case study, as shown in Figure 7, does not include all the details of the building and suffers from low quality. Additionally, the model is not complete and misses parts of the building. For example, the opposite side of the model was not completely restored by SFM. This is because adequate photos from the opposite side could not be taken due to the problem with the photographing location. The photos that are taken from a distance and include the main features of the building are more efficient for the SfM. However, in the dense urban areas or if buildings are occluded by trees, such photos cannot be taken which influence the results. If using SfM technique is not feasible due to limitations in providing photos, other modelling method can be utilized to include the $3 \mathrm{D}$ object in the system.

Additionally, the system shows some inaccuracies in terms of locating the landmark when the smartphone is directed to its direction, and displaying the augmented model. We suspect this error is due to the inaccuracy of the GPS positioning in indoor environments as well as the errors related to the internal digital compass and the gyroscope. The problem of positioning accuracy can be tackled by utilizing more accurate indoor Real-Time Location Tracking Systems (RTLS) instead of GPS.

\section{CONCLUSION AND FUTURE WORK}

The paper presented a method to facilitate way-finding for pedestrians in underground facilities and dense urban environments. The AR-based application overlays the $3 \mathrm{D}$ model of a chosen landmark on the live camera feed of the user's handheld. The augmentation provides the user with the ability to see invisible landmarks, which helps him/her with way-finding intuitively. The SfM technique is utilized to create the model of landmarks from available photos. The case study shows that the 3D models can be added to the video stream when the handheld is directed towards the landmark. The projected model can also be accompanied by navigation information.

The accuracy of the system can be improved by utilizing more accurate RTLS methods (such as, UWB, RFID, and Wireless LAN). Additionally, the detailed investigation on the sources of error and the improvement of the accuracy is considered a future work for this research. Regarding the quality of the 3D model created by SfM, it is suggested to provide aerial photographs. However, other methods can be used to create accurate 3D models. The performance of various software applications to create high density models should also be evaluated. Moreover, the ability to visualize multiple landmarks, add relative distance between the user and the landmarks to the handheld screen and embedding the perspective to the views are considered future steps to complete the prototype system. The case study is planned to be implemented in a larger underground facility in order to validate the benefits of the proposed system.

\section{REFERENCE}

Agarwal, S., Snavely, N., Simon I., Seitz, S., Szeliski, R., 2011. Building Rome in a day, Communications of the ACM, Vol.54 Issue 10, October 2011, pp.105-112.

Agisoft LLC. 2006. Report on Agisoft "Agisoft PhotoScan" http://www.agisoft.com/, (7 April. 2015).

Dellaert, F., Seitz, S., Thorpe, C., and Thrun, S., 2000. Structure from Motion without Correspondence, IEEE Computer Society Conference on Computer Vision and Pattern Recognition, Vol.2, pp.557-564.
Furubayashi, S., Yabuki, N., Fukuda,T., 2013. A data model for Checking Direvtion Singnage at Railway Stations, Proceedings of the First International Conference on Civil and Building Engineering Informatics, ICCBEI 2013, November 7-8, Tokyo, Japan, pp.167-174.

Furukawa, Y., Ponce, J., Team, W., and Inria, E.N.S. 2010. Accurate, Dense, and Robust Multi-View Stereopsis, IEEE Trans. Pattern Anal. Mach. Intell. Vol. 32, Issue 8, pp.1362-1376. Junejo, I.N., Foroosh, H., 2008. GPS coordinate estimation from calibrated cameras, Pattern Recognition, ICPR 2008. 19th International Conference on, pp.1-4.

Kameda, Y., Takemasa, T., Ohta, Y., 2004. Outdoor SeeThrough Vision Utlizing Surveilillance Cameras, Proceedings of the Third IEEE and ACM international Symposium on Mixed and Augmented Reality, ISMAR 2004, pp.213-218.

Metaio Inc. 2013. Report on the metaio "metaio Developer Portal", http://dev.metaio.com/sdk/tutorials/hello-world/ (14 Feb. 2015).

Moulon, P., 2012. OpenMVG: "open Multiple View Geometry", http://imagine.enpc.fr/ moulonp/openMVG/index.html, (12April. 2015).

Schall, G., Mendez, E., Kruijff, E., Veas, E., Junghanns, S., Reitinger, B., Schmalstieg, D. 2009. Handheld Augmented Reality for underground infrastructure visualization, Pres Ubiquit Comput, 13: pp.281-291.

Snavely, N., Seitz, S.M., and Szeliski, R. 2006. Photo Tourism : Exploring Photo Collections in 3D, SIGGRAPH '06 ACM SIGGRAPH 2006 Pap., vol. 1, no. 212, pp. 835-846.

TagCast Inc. 2014. Report on TagCast "Case study - Provide TAGCAST for a large experiment lead by Land, infrastructure and Transportation Ministry of Japan -", https://play.google.com/store/apps/details?id=jp.tagcast.shibuya .navi\&hl=ja (18 Apr. 2015).

Tanighuchi, Y., Aida. S., Yoshinaka, S., Takizawa, A., 2014. Study on Tsunami Evacuation Plan at the Underground Mall in front of Osaka Station, Summaries of technical papers of annual meeting 2014, pp.147-148.

Tardieu, J., Susini, P., Poisson, F., Kawakami, H., and McAdams, S., 2009. The design and evaluation of an auditory way-finding system in a train station, Appl. Acoust., vol. 70, no. 9, pp. 11831193.

Yamauchi, S. 2012. Research on the spatial perception and the guidance method in underground space, Hosei University graduate school design engineering graduate course bulletin, Vol.1. 